\title{
Онтологическая модель иифрового паспорта изделия приборостроительной отрасли
}

\author{
Ю.В. Донеикая 1, к.т.н., доиент, donetskaya_julia@mail.ru \\ E.B. Tкачева 1, acnupaнm, tkacheva@itmo.ru \\ A.A. Токмаков ${ }^{1}$, аспирант, uw5fl.2@yandex.ru \\ ${ }_{1}$ Санкт-Петербургский национальный исследовательский университет \\ информаиионных технологий, механики и оптики (Университет ИТМО), \\ факультет безопасности информаиионных технологий, \\ г. Санкт-Петербург, 197101, Россия
}

В статье на основании результатов анализа аспектов информационного взаимодействия между предприятиями на различных этапах жизненного цикла изделия сформулированы проектные альтернативы компонентов цифрового паспорта изделия. Разработана онтологическая модель цифрового паспорта изделия для приборостроительной отрасли с использованием стандартных элементов метамоделей - объектов, отношений и функций.

В процессе разработки сформированы перечни проектно-производственных процедур в соответствии с этапами жизненного цикла изделия, а также атрибуты и классы данных предметной области. Описаны атрибуты и свойства классов (подклассов) и ограничения на их значения и типы данных. Определены информационные объекты онтологической модели, включающей все возможные варианты описаний компонентов, обобщенных для приборостроительной отрасли. Классификация терминов предметной области представлена иерархическими связями согласно принципу наследования объектно-ориентированного подхода. Классы модели определены множествами терминов предметной области с использованием системы Protégé.

Разработанная онтологическая модель содержит полную структуру данных о разрабатываемом на предприятии изделии с соответствующими процедурами для отрасли приборостроения. Перечень формируемых описаний компонентов цифрового паспорта на конкретном предприятии определяет возможные варианты содержания проектных решений. В качестве примера реализации приведен демонстрирующий алгоритм использования предложенной онтологической модели на предприятии, внедряющем цифровой паспорт изделия для управления внутренними процессами организации производства с выполнением всех этапов жизненного цикла изделия.

Полученная модель может быть передана любому другому предприятию и отрасли в рамках реализации информационных структур взаимодействия при выполнении проектных работ.

Ключевые слова: онтология, цифровой паспорт, приборостроение, жизненный цикл изделия.

Современная радиоэлектронная аппаратура является результатом сложного наукоемкого процесса разработки изделий, требующего хранения и анализа массивных объемов данных о каждом этапе разработки и жизненного ицикла изделия (ЖЦИ) для обеспечения высокого качества и надежности итоговой продукции. Организация хранения информации о разрабатываемом электронном изделии возможна за счет внедрения цифрового паспорта, представляющего собой сложную систему математических моделей [1]. Сегодня внедрение цифрового паспорта необходимо для унификации и стандартизации процедур представления информации об изделии на самом предприятии, а также для обеспечения взаимодействия отделов предприятия независимо от того, какие системы внедряются или уже внедрены и эксплуатируются.

Существующие подходы к обеспечению автоматизации этапов ЖЦИ и организации взаимодействия между отделами предприятия нацелены на решение проблемы интегрирования данных между системами проектирования, но не обеспечивают поддержку эффективного взаимодействия различных предприятий без привязки к определенным системам проектирования. Так, в работе [2] предлагается представить разработку сложного технического проекта как отдельный процесс с применением вспомогательных программных инструментов и сред. Данный подход должен сократить жизненный цикл разработки и снизить за счет этого расходы, но для введения вспомогательных систем потребуется допол- 
нительное согласование используемых инструментов, что увеличивает нагрузку на старте проекта. В статье [3] предложен подход к интеграции между инструментами системного проектирования с помощью преобразования структуры данных на примере инструмента моделе-ориентированной системной инженерии PolarSys Capella и PLMплатформы T-FLEX DOCs. Сегодня на рынке не существует оптимального решения в виде единой информационной системы для проектирования и разработки сложных объектов приборостроительной отрасли, которая поддерживала бы связь между подходом моделирования объектов системной инженерии и концепцией управления жизненным циклом продукта, а также предоставляла бы единое пространство для работы с полной цифровой моделью изделия без использования сторонних ресурсов в виде вспомогательного ПО, хранилища данных (справочников), временных затрат. Предложенный авторами метод применим к конкретным средам проектирования, что не отражает полного покрытия всех систем автоматизированного проектирования.

В большинстве случаев в процессе исполнения договоров заказчиков предприятие работает не обособленно, а сотрудничая с другими предприятиями, одновременно выполняя работы на различных этапах ЖЦИ, поэтому можно столкнуться с конфликтом взаимодействия различных систем проектирования и структуры данных на отдельных предприятиях. Чтобы избежать этого, на этапе заключения договоров необходимо согласовывать структуры данных при взаимодействии с другими предприятиями, а это приводит к увеличению сроков согласования и подписания договоров. Для исключения лишних временных затрат на согласование структуры данных и используемых систем проектирования требуется унифицированная структура данных об электронном изделии со сквозным фиксированием изменений на каждом этапе ЖЦИ [4]. В настоящем исследовании представлены результаты разработки онтологической модели цифрового паспорта изделия для приборостроительной отрасли.

\section{Задача разработки онтологии цифрового паспорта}

Обобщение данных об электронном описании изделия возможно за счет подробной формализации путем построения концептуальной схемы - онтологии. Онтология циф- рового паспорта электронного описания изделия содержит данные об изделии (в виде информационных объектов цифрового паспорта) и проектно-производственных процедурах, выполняемых на этапах ЖЦИ [5].

Проектирование онтологии предполагает первоначальное определение ее предметной области, терминов и классов, масштабов и границ, организацию иерархии терминов, формирование атрибутов и свойств терминов, определение значений. Построенная онтологическая модель будет максимально полно и достоверно воспроизводить ключевые аспекты рассматриваемой предметной области - электронные объекты цифрового паспорта изделия, используя стандартные элементы метамоделей - классы, атрибуты, отношения и т.д. [6-9].

Формальная модель онтологии описывается выражением $O=\langle T, A, R\rangle$, где $T$ - множество терминов предметной области онтологии; $A$ - множество атрибутов конкретного термина, описывающих классы онтологии; $R$ - множество отношений между терминами.

Описание множеств атрибутов и отношений между терминами и классами возможно введением дополнительных кортежей: $O=$ $=\left\langle T, R_{T}, R\right\rangle$, где $R_{T}$ - отношение «род-вид» между терминами и $A=\left\langle N_{A}, T_{P}, V\right\rangle$, где $N_{A}-$ наименование атрибута; $T_{P}-$ тип атрибута; $V$ - значение атрибута.

Таким образом, в настоящей статье онтология $O$ - это кортеж, состоящий из множества классов $(T)$, множества атрибутов $(A)$, матрицы сопоставлений $(P)$, матрицы смежности $(R)$ и заданный в виде $O=\langle T, A, P, R\rangle$, где $T=\left(t_{1}, t_{2}, \ldots, t_{n}\right)-$ множество $n$ классов онтологии, определяющих проектно-производственные процедуры и информационные объекты на всех этапах ЖЦИ; $A=\left(a_{1}, a_{2}, \ldots, a_{p}\right)$ множество $p$ атрибутов, описывающих классы онтологии в системах управления; $P$ - матрица сопоставлений атрибутов классам онтологии; $R$ - матрица смежности, описывающая взаимосвязи между классами.

Такое представление модели позволяет сформировать граф базовой онтологии $[10,11]$, вершинами которого являются элементы множеств $T$ и $A$, а ребрами $-P$ и $R$, что дает возможность использовать для формирования онтологии методы теории графов.

\section{Формирование множества классов онтологии и матрицы смежности}

При анализе предметной области для каждого этапа ЖЦИ были сформулированы пе- 
речни проектно-производственных процедур, которые выполняются сотрудниками предприятия в ходе проектной работы. Результатом выполнения каждой такой процедуры являются данные об изделии. Под данными в настоящей работе понимаются конструкторская программная или технологическая электронная документация, в том числе иная техническая документация в виде производ- ственных журналов замечаний, производственных журналов заводских номеров изделия и его составных частей, журналов предъявления продукции в отдел технического контроля (ОТК) и прочее.

Для каждого этапа ЖЦИ были сформированы пары «данные об изделии-проектнопроизводственная процедура» (табл. 1). Следует заметить, что перечни производствен-

\section{Проектные альтернативы компонентов цифрового паспорта изделия в отрасли приборостроения}

Design alternatives for digital passport components in the instrument-making industry

Таблица 1

\begin{tabular}{|c|c|c|}
\hline Этап ЖЦИ & Проектно-производственная процедура & Формируемый информационный объект \\
\hline \multirow[t]{3}{*}{$\begin{array}{l}\text { Заключение } \\
\text { договора }\end{array}$} & $\begin{array}{l}\text { Рассмотрение и согласование заявки на разра- } \\
\text { ботку или поставку продукции }\end{array}$ & $\begin{array}{l}\text { Объект «Документ» вида «Заявка на разработку»; } \\
\text { «Заявка на поставку продукции» } \\
\end{array}$ \\
\hline & $\begin{array}{l}\text { Подготовка, регистрация и подписание дого- } \\
\text { вора с заказчиком }\end{array}$ & Объект «Документ» вида «Договор с заказчиком» \\
\hline & $\begin{array}{l}\text { Регистрация и подписание договоров } \\
\text { с соисполнителями }\end{array}$ & Объект «Документ» вида «Договор с соисполнителем» \\
\hline \multirow[t]{7}{*}{ Разработка } & $\begin{array}{l}\text { Разработка, согласование и утверждение } \\
\text { укрупненного графика работ }\end{array}$ & $\begin{array}{l}\text { Объект «Документ» вида «Унифицированное задание»; } \\
\text { объект «Изделие» вида «Комплекс» }\end{array}$ \\
\hline & \begin{tabular}{|l|} 
Управление актуализацией данных \\
о покупных комплектующих изделиях (ПКИ)
\end{tabular} & $\begin{array}{l}\text { Объект «Изделие» вида «Деталь»; } \\
\text { объект «CAD-Документ» вида «Модель детали» }\end{array}$ \\
\hline & $\begin{array}{l}\text { Разработка и изменение данных печатной } \\
\text { платы }\end{array}$ & $\begin{array}{l}\text { Объект «Изделие» вида «Деталь»; «Сборочная единица»; } \\
\text { объект «САD-Документ» вида «Модель детали»; } \\
\text { «Модель сборочной единицы»; «Чертеж ...»; объект } \\
\text { «Документ» вида «Схема ...»; «Перечень элементов»; } \\
\text { «Спецификация» }\end{array}$ \\
\hline & Разработка и изменение конструкции прибора & $\begin{array}{l}\text { Объект «Изделие» вида «Деталь»; «Сборочная единица»; } \\
\text { «Комплект»; объект «САD-Документ» вида «Модель } \\
\text { детали»; «Модель сборочной единицы»; «Чертеж ...»; } \\
\text { объект «Документ» вида «Паспорт»; «Таблица } \\
\text { проверок»; «Спецификация»; «Ведомость ...»; } \\
\text { «Технические условия»; «Программа и методика } \\
\text { испытаний»; «Инструкция ...» }\end{array}$ \\
\hline & Разработка и изменение ПО прибора & $\begin{array}{l}\text { Объект «Изделие» вида «Компонент»; «Комплекс»; } \\
\text { «Текст программы»; «Спецификация» }\end{array}$ \\
\hline & $\begin{array}{l}\text { Разработка и изменение эксплуатационной } \\
\text { документации }\end{array}$ & $\begin{array}{l}\text { Объект «Документ» вида «Паспорт»; «Формуляр»; } \\
\text { «Инструкция ...»; «Руководство по эксплуатации»; } \\
\text { «Этикетка»; «Ведомость ...»; «Каталог изделия»; } \\
\text { «Спецификация» }\end{array}$ \\
\hline & $\begin{array}{l}\begin{array}{l}\text { Согласование, утверждение и сдача комплекта } \\
\text { рабочей конструкторской документации } \\
\text { в архив }\end{array} \\
\end{array}$ & Объекты «CAD-Документ»; «Документ» \\
\hline \multirow[t]{5}{*}{$\begin{array}{l}\text { Подготовка } \\
\text { производства }\end{array}$} & $\begin{array}{l}\text { Разработка и изменение технической } \\
\text { документации на изделие }\end{array}$ & $\begin{array}{l}\text { Объект «Изделие» вида «Деталь»; «Сборочная единица»; } \\
\text { «Технологическая оснастка»; «Спецификация»; объект } \\
\text { «САD-Документ» вида «Управляющая программа»; } \\
\text { «Модель детали»; «Модель сборочной единицы»; } \\
\text { «Чертеж ...»; объект «Документа» вида «Карта } \\
\text { техпроцесса»; «Технологическая инструкция»; «Типовой } \\
\text { технологический процесс» }\end{array}$ \\
\hline & $\begin{array}{l}\text { Запись замечаний и решений при проведении } \\
\text { технологической подготовки производства } \\
\text { и при планировании закупок } \\
\end{array}$ & $\begin{array}{l}\text { Объект «Замечание» вида «Замечание от } \\
\text { технологического отдела»; «Замечание от службы } \\
\text { снабжения» }\end{array}$ \\
\hline & $\begin{array}{l}\text { Подготовка и регистрация договора на по- } \\
\text { ставку ПКИ и материалов }\end{array}$ & Объект «Документ» вида «Договор с поставщиком» \\
\hline & $\begin{array}{l}\text { Фиксирование и регистрация поступления } \\
\text { партии ПКИ, материалов и продукции } \\
\text { межзаводской кооперации }\end{array}$ & $\begin{array}{l}\text { Объект «Документ» вида «Рекламационный акт»; } \\
\text { объект «Экземпляр изделия» }\end{array}$ \\
\hline & $\begin{array}{l}\text { Формирование производственного плана } \\
\text { изготовления продукции }\end{array}$ & «Унифицированное задание» \\
\hline \multirow[t]{3}{*}{ Производство } & $\begin{array}{l}\text { Проверка ПКИ, материалов и продукции } \\
\text { межзаводской кооперации при выдаче со } \\
\text { склада }\end{array}$ & $\begin{array}{l}\text { Объект «Экземпляр изделия»; объект «Документ» вида } \\
\text { «Рекламационный акт» }\end{array}$ \\
\hline & $\begin{array}{l}\text { Фиксирование составов изготавливаемых } \\
\text { сборочных единиц }\end{array}$ & Объект «Экземпляр изделия» \\
\hline & $\begin{array}{l}\text { Запись замечаний и решений в процессе про- } \\
\text { изводства деталей и сборочных единиц изде- } \\
\text { лия }\end{array}$ & Объект «Замечание» вида «Замечание от производства» \\
\hline
\end{tabular}


ных процедур зависят только от этапа ЖЦИ, управление которым реализуется на отдельном предприятии.

Таким образом, данные об изделии представляются как информационные объекты, виды которых определяются эксплуатируемыми на предприятии САПР, с возможностью использовать, описывать и хранить данные с каждой ревизией документа. Содержательной частью информационных объектов являются файлы конструкторской и технологической документации, а реквизитной - атрибуты, определяемые видом используемых средств ПО и систем (СЭД, ERP, PDM, MES или QMS) соответственно [12]. Одним из способов создания такой структуры является построение онтологической модели цифрового паспорта изделия. Как и различные БД, онтология, несомненно, является основой многих приложений, специфичных для предметной области [10]. В последние десятилетия растет признание необходимости создания явной спецификации того, как знания в предметной области формируются, хранятся и передаются. Онтология определяется как схематичная структура, используемая для обмена знаниями между пользователями и программами [13]. Более формально онтология определяется как спецификация словаря терминов и атрибутов, связывающих их [11]. Следовательно, онтологии позволяют выражать сложные взаимоотношения внутри предметной области, а установление отношений между терминами понять концепт не только по его атрибутам, но и по положению относительно остальной онтологии.
Необходимым шагом процесса проектирования онтологии является формирование множества классов предметной области, классификация которых может быть представлена иерархическими связями, используя принцип наследования объектно-ориентированного подхода (рис. 1). Классы онтологии определяются множествами терминов предметной области в процессе формирования подмножеств базовой онтологии с использованием системы Protégé.

Деление классов онтологии цифрового паспорта на подклассы позволяет конкретизировать виды хранимых и обрабатываемых данных о разрабатываемом изделии и проектно-производственных процедурах в соответствии с этапами жизненного цикла (см. http://www.swsys.ru/uploaded/image/2021-4/ 2021-4-dop/13.jpg).

На данном этапе проектирования онтологии формируется матрица смежности с учетом видов отношений между классами $R$ : $R=\left|r_{i j}\right|$, где

$$
r_{i j}=\left\{\begin{array}{l}
1, \text { если классы } t_{i} \text { и } t_{j} \text { связаны между собой } \\
\text { отношениями, } \\
0, \text { если классы } t_{i} \text { и } t_{j} \text { не связаны между } \\
\text { собой отношениями, }
\end{array}\right.
$$

$i, j=\overline{1, n}$.

Отношение «формируется» образует пары «информационный объект-проектно-производственная процедура», являющиеся компонентами цифрового паспорта. Отношение «описывается» задает связи между объектами «Изделие» или объектами «Экземпляр» и соответствующими им объектами «Документ»

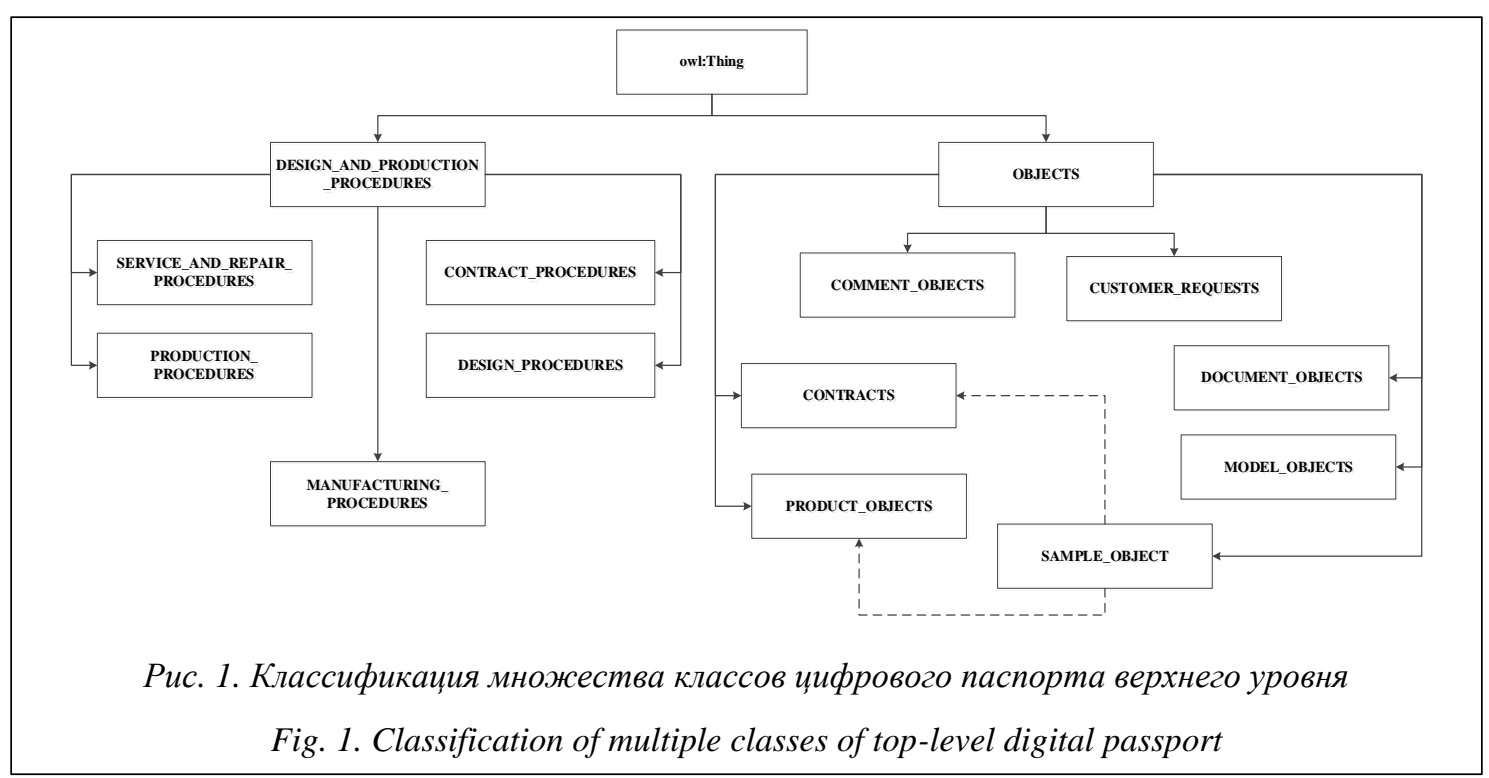


и/или «СAD-Документ». Отношение «состоит из» задает связь различных видов объектов «Изделие» или объектов «Экземпляр» между собой. Отношение «ссылается на» устанавливает взаимосвязи между объектами.

\section{Формирование множества атрибутов онтологии и матрицы сопоставлений}

На следующем этапе проектирования онтологической модели цифрового паспорта определяются атрибуты и свойства классов (подклассов) и ограничения на их значения и типы данных. Атрибуты могут быть сгруппированы по видам систем (табл. 2). Таким образом, классы онтологии описываются перечнями атрибутов, формируемыми средствами СЭД, ERP, PDM, MES, QMS.

Локальные требования предприятий к содержанию цифровых паспортов изделий определяют перечень описаний компонентов формируемых цифровых паспортов, позволяя не использовать весь перечень, предусмот-

\section{Перечень атрибутов в онтологической модели цифрового паспорта}

Таблица 2

The list of attributes in the ontological model of a digital passport

Table 2

\begin{tabular}{|c|c|c|}
\hline Наименование атрибута & Вид системы & Атрибут в системе Protégé \\
\hline номер заявки & СЭД & request_number \\
\hline вид изделия & СЭД, ERP, PDM & product_type \\
\hline номер договора (дополнения к договору) & СЭД, ERP, PDM & contract_number \\
\hline наименование заказчика & СЭД, ERP, PDM & custumer_name \\
\hline реквизиты заказчика & СЭД, ERP, PDM & customer_bank_data \\
\hline наименование соисполнителя & СЭД, ERP, PDM & co-contractor_name \\
\hline реквизиты соисполнителя & СЭД, ERP, PDM & co-contractor_bank_data \\
\hline наименование поставщика & СЭД, ERP, PDM & supplier_name \\
\hline реквизиты поставщика & СЭД, ЕRP, PDM & supplier_bank_data \\
\hline номер графика & ERP, PDM & task_number \\
\hline объем работ & ERP, PDM & scope_of_tasks \\
\hline дата начала работ & ERP, PDM & start_of_tasks \\
\hline дата окончания работ & ERP, PDM & end_of_tasks \\
\hline обозначение & СЭД, ERP, PDM & object_number \\
\hline наименование & СЭД, ERP, PDM & object_name \\
\hline представление & PDM & product_view \\
\hline версия & PDM & version \\
\hline состояние & PDM & state \\
\hline наименование изготовителя & ERP, PDM, MES & executor_name \\
\hline габариты изделия & PDM & product_size \\
\hline масса изделия & PDM & product_mass \\
\hline ограничительный признак & PDM & remark_state \\
\hline вид документа & PDM & document_type \\
\hline вид модели & PDM & model_type \\
\hline первичная применяемость & PDM & first_used_number \\
\hline формат & PDM & document_format \\
\hline количество листов документа & PDM & document_pages \\
\hline литера & PDM & used_type \\
\hline номер решения & PDM & number_of_solution \\
\hline дата утверждения решения & PDM & date_of_solution \\
\hline роль лица & PDM & workflow_role_name \\
\hline Ф.И.О. лица & PDM & user_name \\
\hline дата подписания & PDM & signature_date \\
\hline номер замечания & PDM, QMS & comment_number \\
\hline содержание замечания & СЭД, PDM, QMS & comment_description \\
\hline предлагаемое решение & СЭД, PDM, QMS & decision_description \\
\hline идентификационный номер & СЭД, PDM, MES, QMS & sample_number \\
\hline признак качества & ERP, PDM, MES & quality_type \\
\hline номер склада & ERP, PDM, MES & stogare_number \\
\hline номер цеха & ERP, PDM, MES & plant_number \\
\hline изготовитель экземпляра & ERP, PDM, MES & executor_name \\
\hline результат операции & MES & operation_result \\
\hline результат контроля качества & MES & quality_result \\
\hline количество экземпляров & PDM, MES & sample_quantity \\
\hline причина несоответствия качества & ERP, PDM, MES, QMS & quality_reason \\
\hline дата отгрузки & ERP, PDM & execution_date \\
\hline
\end{tabular}


ренный онтологической моделью. Формируется матрица сопоставлений атрибутов классам онтологии $P$ :

$$
\begin{gathered}
P=\left|p_{i j}\right|, \text { где } \\
p_{i j}=\left\{\begin{array}{c}
<t_{i}, a_{j}>, \text { если атрибут } a_{j} \text { описывает } \\
\text { класс } t_{i}, \\
<0,0>, \text { если атрибут } a_{j} \text { не описывает } \\
\text { класс } t_{i},
\end{array}\right. \\
i=\overline{1, n}, j=\overline{1, p} .
\end{gathered}
$$

Сами перечни формируемых описаний компонентов цифровых паспортов на предприятиях определяют множество атрибутов, описывающих тот или иной класс онтологии. Они соответствуют видам систем управления, средствами которых может быть реализовано управление на этапах ЖЦИ.

\section{Онтологическая модель цифрового паспорта изделия}

Онтологическая модель цифрового паспорта (фрагмент приведен на рисунке 2) является результатом формулирования списка терминов, их атрибутов и свойств. При этом разработанная модель, обобщенная для области приборостроения, включает в себя все возможные варианты описаний компонентов с использованием средств СЭД, ERP, PDM, $\mathrm{MES}, \mathrm{QMS}$. Более того, онтология содержит полную структуру данных о разрабатываемом изделии с соответствующими процедурами для приборостроения.

Онтология представлена кортежем, элементами которого являются множества классов и атрибутов классов онтологии, а также матрицы распределения и смежности. Данная модель является базовой онтологией цифрового паспорта изделия, ее можно использовать для формирования содержания цифрового паспорта изделия и генерации проектных решений посредством выполнения операции извлечения суб-онтологий.

\section{Пример реализации}

В качестве примера реализации настоящей онтологической модели рассматривается предприятие, деятельность которого направлена на разработку и поставку изделий приборостроительной отрасли. Предприятие

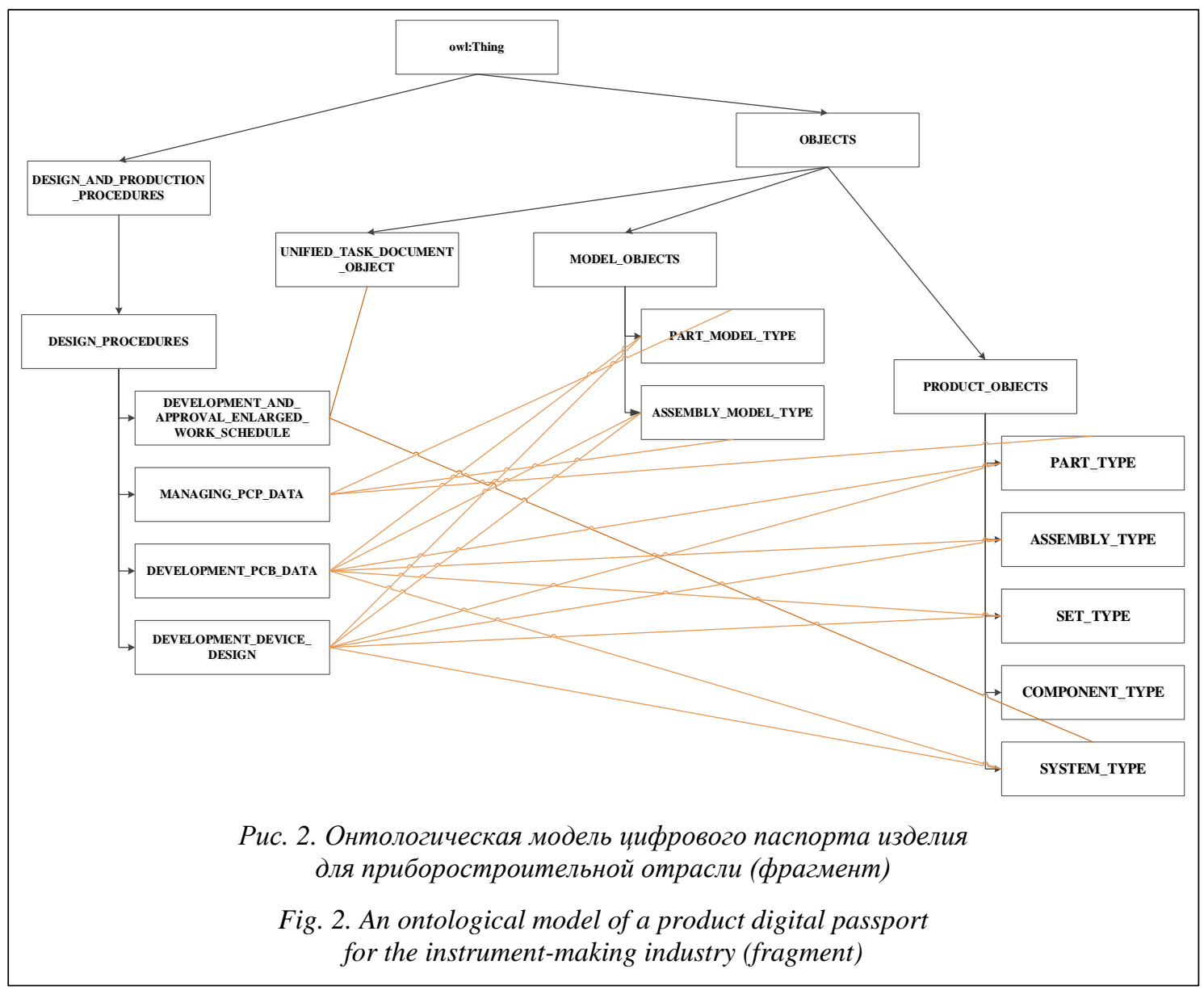


внедряет цифровой паспорт изделия для управления внутренними процессами организации производства с выполнением всех этапов ЖЦИ: заключение договоров, разработка, подготовка к производству, производство, передача в эксплуатацию, поставка изделия, в том числе ремонт и утилизация. Разработанная онтологическая модель отрасли может быть использована как в полном виде, так и выборочно, задействовав отдельные атрибуты, классы и данные базы знаний. Для организации электронного взаимодействия внедряются система Windchill PLM (Product Lifecycle Management) и Корпоративная информационная система «Галактика ERP». Этапы жизненного цикла и перечень систем определены в рамках требований, предъявляемых к содержанию цифрового паспорта изделия.

Анализ требований выполняется на основе известных методов формирования экспертных оценок [14, 15], результатом применения которых является формирование перечней информационных объектов и проектно-производственных процедур, управляемых средствами цифрового паспорта. Для определения структуры цифрового паспорта электронного описания изделия на предприятии используется метод извлечения данных из онтологической модели на основе перечня информационных объектов [16], который фокусируется на извлечении суб-онтологии из базовой и замене онтологий в облаке. Используя этот подход, предприятие извлекает онтологию на основе перечня информационных объектов и проектно-производственных процедур, полученных при анализе экспертных оценок.

\section{Заключение}

Необходимо отметить, что была построена онтологическая модель для цифрового паспорта электронного изделия, каждому объекту указанных информационных систем поставлены в соответствие элемент онтологии, связи между информационными объектами, что позволило сформировать структуру представления данных об изготавливаемых предприятием изделиях. Полученная структура может быть передана любому другому предприятию и отрасли в рамках реализации информационных структур взаимодействия при выполнении проектных работ. Приведен пример, демонстрирующий алгоритм реализации предложенной онтологической модели на предприятии приборостроительной отрасли.

\section{Лuтература}

1. Adisorn T., Tholen L., Götz T. Towards a digital product passport fit for contributing to a circular economy. Energies, 2021, vol. 14, no. 8, art. 2289. DOI: 10.3390/en14082289.

2. Korolev A.S., Shamanin A.Y. The use of formal methods of verification and validation in NPP design. Proc. XI Int. Conf. MLSD, 2018, pp. 1-4. DOI: 10.1109/MLSD.2018.8551837.

3. Королев А.С., Кюрчева С.Г. Проблематика и описание подхода к интеграции инструментов системного проектирования // INJOIT. 2021. Т. 9. № 8. С. 16-22.

4. Афанасьев В.Б. Онтологическое проектирование автоматизированной информационной системы поддержки качества продукции предприятия // Изв. ТулГУ. Технические науки. 2020. № 10. С. $12-22$.

5. Донецкая Ю.В. Метод формирования электронного описания изделия // Научно-технический вестник информационных технологий, механики и оптики. 2008. № 46. С. 40-43.

6. Боргост Н.М., Шустова Д.В., Гиматдинова С.Р. Иерархические и ассоциативные связи между терминами в тезаурусе на примере словаря проектанта // Вестн. СГАУ. 2012. № 2. С. 228-236.

7. Кравченко Ю.А., Новиков А.А., Марков В.В. Метод создания онтологии предметной области на основе глоссария // Изв. ЮФУ. Технические науки. 2015. № 6. С. 158-168.

8. Шустова Д.В. Подход к разработке семантических основ информационных систем для проектирования и производства авиационной техники // Онтология проектирования. 2015. Т. 5. № 1. C. $70-84$.

9. Donetskaya J., Gatchin Yu. A generalization of approaches to creating a digital passport supporting the stages of the electronic product life cycle and the features of the formation of design decisions based on it. Industry 4.0, 2020, vol. 5, no. 3, pp. 101-104.

10. Flahive A. et al. A methodology for ontology update in the semantic grid environment. Concurrency and Computation: Practice and Experience, 2015, vol. 27, no. 4, pp. 782-808. DOI: 10.1002/cpe.2841. 
11. Flahive A., Taniar D., Rahayu W. Ontology as a Service (OaaS): a case for sub-ontology merging on the cloud. The J. of Supercomputing, 2013, vol. 65, no. 1, pp. 185-216. DOI: 10.1007/s11227-011$0711-4$.

12. Otte J.N. et al. An ontological approach to representing the product life cycle. Applied Ontology, 2019, vol. 14, no. 2, pp. 179-197. DOI: 10.3233/AO-190210.

13. Preece A. et al. An ontology-based approach to handling information quality in e-Science. Concurrency and Computation: Practice and Experience, 2008, vol. 20, no. 3, pp. 253-264. DOI: 10.1002/ cpe. 1195 .

14. Данелян Т.Я. Формальные методы экспертных оценок // Статистика и экономика. 2015. № 1. С. $183-187$.

15. Финогенко И.А., Дьякович М.П. Метод анализа иерархий и построение интегральных показателей сложных систем // Вестн. Тамбовского университета. Сер.: Естественные и технические науки. 2017. Т. 22. № 6-1. С. 1335-1340. DOI: 10.20310/1810-0198-2017-22-6-1335-1340.

16. Flahive A., Taniar D., Rahayu W. Ontology as a Service (OaaS): extracting and replacing sub-ontologies on the cloud. Cluster computing, 2013, vol. 16, no. 4, pp. 947-960. DOI: 10.1007/s10586-0120231-x.

Software \& Systems

DOI: $10.15827 / 0236-235 X .136 .608-616$
Received 07.07.21, Revised 04.10.21

2021, vol. 34, no. 4, pp. 608-616

\section{An ontological model of a product digital passport for the instrument-making industry}

J.V. Donetskaya ${ }^{1}$,Ph.D. (Engineering), Associate Professor, donetskaya_julia@mail.ru

E.V.Tkacheva ${ }^{1}$, Postgraduate Student, tkacheva@itmo.ru

A.A. Tokmakov ${ }^{1}$, Postgraduate Student, uw5fl.2@yandex.ru

${ }^{1}$ The National Research University of Information Technologies,

Faculty of Information Technology Security, Mechanics and Optics, St. Petersburg, 197101, Russian Federation

Abstract. The paper formulates design alternatives for a product digital passport components in the instrument-making industry based on the results of the analysis of the information interaction between enterprises at various stages of an electronic product life cycle. An ontological model of the knowledge database of an electronic product digital passport for the instrument-making industry has been developed based on the metamodels standard elements - objects, relations and functions.

The development process involved forming the lists of design and production procedures in accordance with electronic product life cycle stages, as well as attributes and data classes of the subject area. The paper describes the properties and attributes of classes (subclasses) and restrictions on their values and data types, determines information objects of the ontological model, which includes all possible options for describing components generalized for the instrument-making industry. The classification of the subject area terms is represented by hierarchical relationships according to the principle of inheritance of the object-oriented approach. Model classes are defined by sets of subject area terms using the Protégé system.

The developed ontological model contains the complete data structure of the product being developed at the enterprise with the corresponding procedures for the instrument-making industry. The list of generated descriptions of the digital passport components at a particular enterprise determines the possible options for the content of design solutions. As a utilization example, there is a demonstrating algorithm for introducing the proposed ontological model to an enterprise in the instrument-making industry, which implements an electronic product digital passport to manage the internal processes of organizing production with the utilization of all stages of an electronic product life cycle.

The resulting structure can be transferred to any other enterprise and industry, as part of the implementation of information structures of interaction when performing design work.

Keywords: ontology, digital passport, electronic product, product life cycle. 


\section{References}

1. Adisorn T., Tholen L., Götz T. Towards a digital product passport fit for contributing to a circular economy. Energies, 2021, vol. 14, no. 8, art. 2289. DOI: 10.3390/en14082289.

2. Korolev A.S., Shamanin A.Y. The use of formal methods of verification and validation in NPP design. Proc. XI Int. Conf. MLSD, 2018, pp. 1-4. DOI: 10.1109/MLSD.2018.8551837.

3. Korolev A.S., Kyurcheva S.G. Problems and description of the approach to integrating systems design tools. INJOIT, 2021, vol. 9, no. 8, pp. 16-22.

4. Afanasyev V.B. Ontological design of an automated information system for supporting the quality of products of the enterprise. Izvestiya TulGU. Technical Sciences, 2020, no. 10, pp. 12-22 (in Russ.).

5. Donetskaya J.V. A method of generating an electronic product description. Sci. Tech. J. Inf. Technol. Mech. Opt., 2008, no. 46, pp. 40-43 (in Russ.).

6. Borgost N.M., Shustova D.V., Gimatdinova S.R. Hierarchical and associative relations between the terms in the thesaurus in a designer's dictionary taken as an example. Vestnik of Samara State University. Aerospace and Mechanical Engineering, 2012, no. 2, pp. 228-236 (in Russ.).

7. Kravchenko Yu.A., Novikov A.A., Markov V.V. Method of creating a domain ontology from glossary. Izvestiya SFedU. Engineering Sci., 2015, no. 6, pp. 158-168 (in Russ.).

8. Shustova D.V. Approach to developing a semantic basis of information systems for aircraft systems design and production. Ontology of Designing, 2015, vol. 5, no. 1, pp. 70-84 (in Russ.).

9. Donetskaya J., Gatchin Yu. A generalization of approaches to creating a digital passport supporting the stages of the electronic product life cycle and the features of the formation of design decisions based on it. Industry 4.0, 2020, vol. 5, no. 3, pp. 101-104.

10. Flahive A. et al. A methodology for ontology update in the semantic grid environment. Concurrency and Computation: Practice and Experience, 2015, vol. 27, no. 4, pp. 782-808. DOI: 10.1002/cpe.2841.

11. Flahive A., Taniar D., Rahayu W. Ontology as a Service (OaaS): a case for sub-ontology merging on the cloud. The J. of Supercomputing, 2013, vol. 65, no. 1, pp. 185-216. DOI: 10.1007/s11227-011-0711-4.

12. Otte J.N. et al. An ontological approach to representing the product life cycle. Applied Ontology, 2019, vol. 14, no. 2, pp. 179-197. DOI: 10.3233/AO-190210.

13. Preece A. et al. An ontology-based approach to handling information quality in e-Science. Concurrency and Computation: Practice and Experience, 2008, vol. 20, no. 3, pp. 253-264. DOI: 10.1002/cpe. 1195.

14. Danelyan T.Ya. Formal methods of expert estimations. Statistics and Economics, 2015, no. 1, pp. $183-187$ (in Russ.).

15. Finogenko I.A., Dyakovich M.P. Method of analysis of hierarchies and construction integrated parameters for multiple systems. Tambov University Reports. Ser.: Natural and Technical Sciences, 2017, vol. 22, no. 6-1, pp. 1335-1340 (in Russ.). DOI: 10.20310/1810-0198-2017-22-6-1335-1340.

16. Flahive A., Taniar D., Rahayu W. Ontology as a Service (OaaS): extracting and replacing sub-ontologies on the cloud. Cluster Computing, 2013, vol. 16, no. 4, pp. 947-960. DOI: 10.1007/s10586-0120231-x.

\section{Для цитирования}

Донецкая Ю.В., Ткачева Е.В., Токмаков А.А. Онтологическая модель цифрового паспорта изделия приборостроительной отрасли // Программные продукты и системы. 2021. Т. 34. № 4. C. 608-616. DOI: 10.15827/0236-235X.136.608-616.

\section{For citation}

Donetskaya J.V., Tkacheva E.V., Tokmakov A.A. An ontological model of a product digital passport for the instrument-making industry. Software \& Systems, 2021, vol. 34, no. 4, pp. 608-616 (in Russ.). DOI: 10.15827/0236-235X.136.608-616. 